International Journal of Modern Physics B

Vol. 33, No. 11 (2019) 1950100 (19 pages)

(C) The Author(s)

DOI: $10.1142 / \mathrm{S} 0217979219501005$

\title{
Analyzing the topological characteristic and key nodes of Chinese air sector network
}

\author{
Guangjian Ren*, Chaoyang $\mathrm{Lu}^{\dagger}$, Jinfu Zhu and Xinyi Liu \\ College of Civil Aviation, Nanjing University \\ of Aeronautics and Astronautics, \\ Nanjing 210016, P. R. China \\ *ZC416418@126.com \\ ${ }^{\dagger}$ chaoyang_lu@163.com
}

Received 5 December 2018

Revised 29 January 2019

Accepted 11 February 2019

Published 7 March 2019

\begin{abstract}
In this paper, an air sector network (ASN) model is established based on the airspace of Chinese mainland. Meanwhile, the ASN is a weighted graph whose nodes are sectors, and its edge weight is the flights between sectors. In addition, a node ranking algorithm is proposed according to the variation coefficient method (VCM). Then, the topological structure of the ASN is analyzed by the complex network theory, simultaneously, the key sectors are ranked through the proposed VCM. The results indicate that the ASN has heterogeneous and assortativity structure characteristics to a certain extent. Finally, the effectiveness of the proposed VCM centrality measure is verified by the susceptibleinfected-removed (SIR) model and Kendall's tau coefficient. Results show that the VCM centrality method is applicable and effective.
\end{abstract}

Keywords: Air sector network; flights; complex network theory; variation coefficient.

PACS number: 59.75.Fb

\section{Introduction}

Airspace is a kind of resource that provides the specific place for aircraft operations. In order to improve the safety and efficiency of air traffic, the airspace is divided into multiple areas, which are called air sectors. Each sector has a group of controllers to provide air traffic control (ATC) services, which guarantees the normal flight of the aircraft.

This is an Open Access article published by World Scientific Publishing Company. It is distributed under the terms of the Creative Commons Attribution 4.0 (CC-BY) License. Further distribution of this work is permitted, provided the original work is properly cited.

$\dagger$ Corresponding author. 
On the other hand, the traffic distribution of each sector is also different. For example, according to the Civil Aviation Administration (CAA) of China (2017), the average daily traffic volume of the top-3 busy sectors is $1.76,1.68$, and 1.51 thousand, respectively. Consequently, the traffic pressure of the sector is also relatively significant.

Besides, each sector does not exist in isolation, but is interconnected by air routes or flights to form a complex transport network. The analysis of the complexity and topological characteristics of the network is conducive to optimizing airspace configuration and improving operation efficiency.

The sectors in airspace constitute a kind of transport network and they can be analyzed by complex network theory. Recently, the study of complex networks applied to air transport has become very broad. $\frac{1}{2}$ Zhang et al. ${ }^{2}$ propose a network model based on the Chinese air route network, then they discuss the network structure and flight conflict characteristics. Besides, Dunn and Wilkinson ${ }^{3}$ study the resilience of air traffic networks and find that the geographical location of airports leaves them vulnerable to spatial hazard, when the traffic networks have fixed air routes. The world airline network model is built in Ref. 4, and the growth characteristics of the model are studied. Meanwhile, Verma et al..$\frac{5}{}$ make a relatively comprehensive analysis of the structural characteristics of the world airline network. In addition, some typical aviation networks such as US airline system, $\underline{6}$ North American airline, ${ }^{7}$ a European airport network ${ }^{8}$ and Australian airport network ${ }^{9}$ are also discussed using complex network theory, results show that they have their own characteristics in terms of operation efficiency and structure, but most of them have small world or scale-free properties. Similarly, the Chinese provincial air transportation network is analyzed as a weighted model in Ref. 10, results demonstrate that the network has small-world and homogeneous features, meanwhile, the distribution of flight flow on the network is heterogeneous. Wen et al. ${ }^{11}$ discuss the Chinese airport network (CAN) as multi-layer networks by complex network method and find that the CAN is not as redundant and robust as the Worldwide airline network under the high-degree targeted attack strategy. Asian air transport network is studied as a complex network model in Ref. 12 by backbone extraction techniques, then the key topological and spatial features are analyzed by complex network theory. The above analysis of the airport networks reflects the different characteristics of different regional networks and they provide a rich reference for further research.

In addition, research on other aspects of air networks has been extensively studied. The vital edges of Chinese air navigation route network are analyzed in Ref. 13, illustrating that the most important edge is not necessarily the edge of the highest topological importance. In Ref. 14, the air navigation point network of fifteen countries is studied and the results show that they all have heterogeneous characteristics. The European airspace as a multi-scale traffic network is discussed in Ref. 15, meanwhile, the community structure of the network obtains an objective research. Roy et al. $\frac{16}{}$ study the vulnerability of the airspace system by some topological 
metrics, from the perspective of the network model. The robustness of the Virgin America air transport network is analyzed in Ref. 17 through the algebraic connectivity. Besides, Lordan et al. $\frac{18}{}$ study the robustness of world air transport network by attacking critical airports and results show that isolating a small number of selected nodes may cause serious problems for the operation of the global network. Sun et al. $\frac{19}{1}$ discuss the temporal evolution of the European air transport system, finding that the air route network is dominated by seasonal changes in summer and winter, however, the airport network is not only affected by summer and autumn, but also by peak and off-peak patterns. The major airports of the world airport network is analyzed in Ref. 20. Moreover, the existence of transition point in Chinese multi-layer air transport networks is proved, which helps to optimize the configuration of the air network. ${ }^{21}$ Sun et al. ${ }^{22}$ study the criticality of nodes in air transport network by three domains and the work gives a better understanding of node criticality in air transport networks.

Furthermore, many centrality measures have been used commonly in recent years. In Refs. 23 and 24, the method for identifying influential nodes in complex networks with community structure is analyzed. Chen et al. ${ }^{25}$ propose a Cluster Rank theory, which takes into account both the number of neighbors and the clustering coefficient. Besides, a centrality measure called hybrid degree centrality (HC) is proposed in Ref. 26 by considering the spreading ability of the nodes and the accuracy of HC is proved by SIR model. Du et al. ${ }^{27}$ propose a centrality measure named TOPSIS and the efficiency and practicability of the TOPSIS are evaluated via the susceptible-infected (SI) model. Moreover, the entropy theory is analyzed in Refs. 28 and 29, then the influential nodes are ranked by the entropy-based approach. Wang et al. ${ }^{30}$ use the efficiency centrality (EffC) to identify influential nodes, results show that the EffC is a feasible and effective centrality measure. Meanwhile, in Ref. 31, a centrality measure based on the analytic hierarchy process (AHP) method is proposed and the validity of AHP is illustrated by the experiments. A special network centrality measure called degree ratio is proposed in Ref. 32, and results show that the metric is valid. Moreover, Pien et al. propose a new index called the relative area index (RAI) in Ref. 33, and the RAI performs well in quantifying the importance of node in the entire air traffic network.

According to the previous analysis, most of the researches are focused on air transport networks, however, there are less researches on airspace networks. In this paper, a weighted network model is established based on the air sectors of Chinese mainland. Then, the topological characteristics of the model are analyzed by the complex network theory and the key sectors are ranked by variation coefficient method (VCM) theory. The effectiveness of the proposed VCM centrality measure is verified by the susceptible-infected-removed (SIR) model and Kendall's tau coefficient. Results show that the VCM centrality method is applicable and effective. The significance of the paper is that it is useful for identifying the influential sectors and providing some theoretical references for optimizing the structure of sectors and improving the efficiency of airspace. 
The rest of this paper is structured as follows. In Sec. 2, the air sector network (ASN) model is built by analyzing the air sector data of Chinese mainland. Then, some topological feature algorithms of complex theory are introduced, meanwhile, a node sorting algorithm based on VCM is proposed in Sec. 3. Next, in Sec. 4, the topological features of ASN are discussed. Key sectors of the ASN are ranked by the proposed VCM in Sec. 5. Finally, Sec. 6 summarizes the conclusions and provides new directions for future research.

\section{ASN Model}

\subsection{Air sector data}

Airspace is the activity area of aircrafts in atmospheric space, which has legal, natural and technical attributes. The airspace is divided into different regions called sectors. Besides, the sectors can be roughly divided into two types: the en-route sectors, which control the trajectory of the aircrafts and terminal control area or the control zones, which manage and guide the process of aircraft take-off and landing. The size and structure of the sector are important for the safety and efficiency of aviation operations.

In addition, sectors are the smallest control units and are directly supervised by the air traffic controllers. According to the flight level distribution of the airspace, the sectors are divided into the high-altitude sectors and the low-altitude sectors. Generally speaking, the projection of some sectors on a two-dimensional plane is identical and these sectors are superimposed on the same level (i.e., ignore the difference in height between the sectors). In this section, we use the database of the Chinese air traffic to investigate the topological properties of the sector network and the key sectors, which can reflect the crowding mechanism of the airspace. Simultaneously, this research can help to master the structural topological characteristics of airspace networks, and be familiar with the characteristics of traffic distribution in air route networks. In this way, it is helpful to find a suitable airspace optimization adjustment scheme, and then take targeted measures to improve airspace utilization efficiency.

On the basis of the Air Traffic Management Bureau (ATMB) of China, the air sectors of Chinese Mainland are shown in Fig. 1.

As shown in Fig. 1, the blue polygons are the air sectors where the solid blue line is the boundary of the sector. Each sector and its neighbors are generally associated with air routes or flight flow, forming an air transport network. According to the statistics of the CAA of China (2017), the average daily traffic of the sectors (top 20) is shown in Table 1.

Table 1 shows that the average daily flight per sector of the top-20 sectors is 1016, and the standard deviation is 306 . Therefore, the busyness of regional sectors is quite different, and the uneven distribution of traffic is prominent. There are thre sectors with more than 1500 flights, accounting for about $2.7 \%$. At the same time, the average daily flights of the top- 20 sectors are greater than 750 . From 


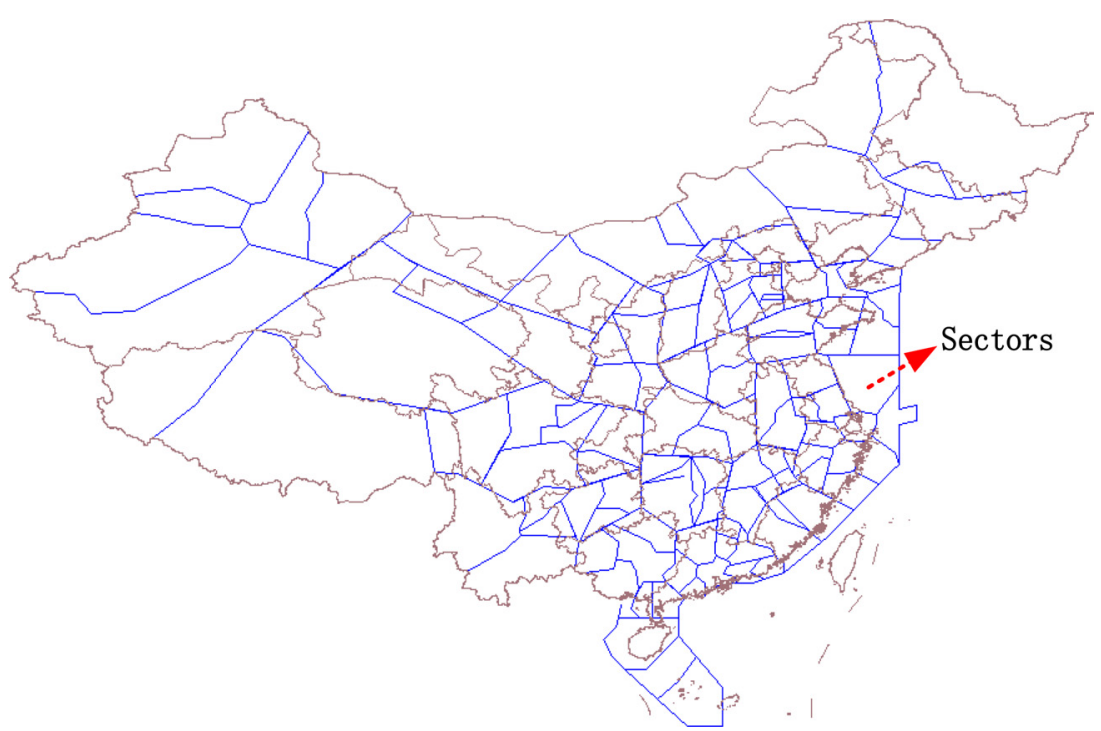

Fig. 1. (Color online) The spatial distribution of sectors in Chinese mainland.

Table 1. The average daily traffic of the top-20 sectors.

\begin{tabular}{cccccc}
\hline Top- $k$ & Sectors & Average daily flights & Top- $k$ & Sectors & Average daily flights \\
\hline 1 & ZBAAAR01 & 1761 & 11 & ZSAMAR02 & 855 \\
2 & ZBAAAR02 & 1682 & 12 & ZBAAAR16 & 851 \\
3 & ZGGGAR05 & 1510 & 13 & ZUUUAR05 & 837 \\
4 & ZBAAAR03 & 1209 & 14 & ZGGGAR15 & 837 \\
5 & ZBAAAR06 & 1161 & 15 & ZSQDAR03 & 836 \\
6 & ZGGGAR13 & 1099 & 16 & ZSSSAR14 & 786 \\
7 & ZSSSAR01 & 1054 & 17 & ZBAAAR12 & 774 \\
8 & ZSSSAR04 & 952 & 18 & ZSAMAR01 & 769 \\
9 & ZGHAAR02 & 940 & 19 & ZSSSAR13 & 768 \\
10 & ZBAAAR04 & 877 & 20 & ZBAAAR05 & 765 \\
\hline
\end{tabular}

the perspective of airspace distribution, the flight volume of some sectors is too large, which is one of the main bottlenecks of airspace capacity limitation, which has an important impact on airspace operation. Thus, identifying critical sectors is beneficial to optimizing the airborne environment.

\subsection{The ASN model}

In this section, we analyze the characteristics of Chinese sector distribution and construct a sector reduction network model. The model construction process is as follows:

(i) Each sector is reduced to a node, and if there is a route between the two nodes, an edge is added. 
(ii) There may be multiple route connections between the two sectors, and multiple routes are concentrated into one edge.

(iii) The high-altitude sector and the low-altitude sector are divided according to different height levels, and if the projection of the sectors on a two-dimensional plane is identical, then they are concentrated into one node during the reduction process.

(iv) The sector network model is an undirected weighted graph and the weight of each edge is the amount of flights between sectors.

According to the above process, the ASN model is shown in Fig. 2.

Figure 2 depicts the ASN of Chinese mainland. In the figure, the solid blue line indicates the edge between the nodes, and the solid circle represents the nodes, that is, the sectors. The larger green circles are the sectors of the top-20 sectors (see Table 1) of the flights, and the red are the other ones. As we have seen, these sectors are interconnected by flights to form a complex network system. Therefore, the problem of operation in the air sectors can be analyzed using complex network theory. The basic topological characteristics of the model can be obtained through complex network theory, as shown in Table 2.

It is shown in Table 2 that the ASN model has 108 nodes and 241 edges, which indicates that the density of the network is 0.042 . Moreover, the average degree of the network is about 4 , illustrating that on average, each sector is directly connected to 4 neighbors. In addition, the average path length is 5.48 , meaning that on average,

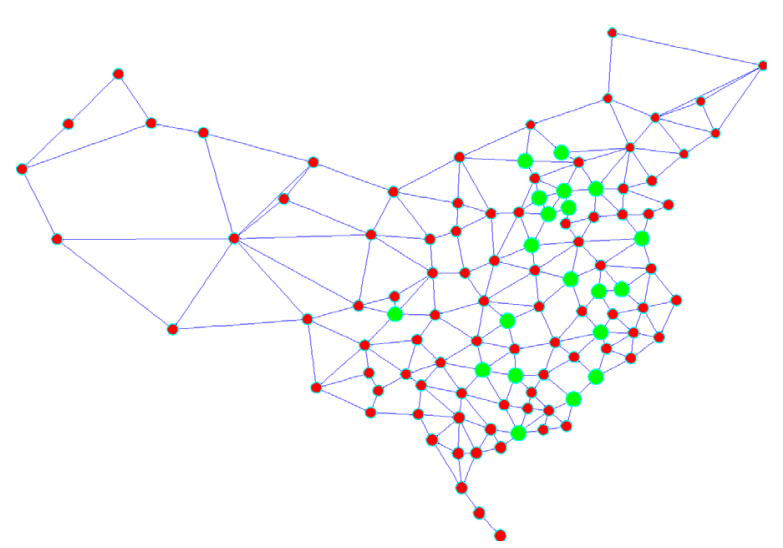

Fig. 2. (Color online) The ASN model. (The figure is a simplified network, which only shows the skeleton of the ASN. Each node contains air route information in one sector and the edge thickness proportional to the edge weights.)

Table 2. The basic topological characteristics of the ASN model.

\begin{tabular}{cccccc}
\hline Parameters & Nodes & Edges & Average degree & Clustering coefficient & Average path length \\
\hline ASN & 108 & 241 & 4.48 & 0.36 & 5.48 \\
\hline
\end{tabular}


each path goes through about 5 nodes. Simultaneously, the clustering coefficient reflects the density of connections between nodes and their neighbors, namely, the frequency of contact between sectors.

\section{Features Analysis Algorithm of ASN}

The ASN is represented as $G(V, E)$ where $V\left(=\left[v_{1}, v_{2}, \ldots, v_{N}\right]\right)$ is the set of nodes and $E\left(=\left[e, e, \ldots, e_{M}\right]\right)$ is the set of edges. Meanwhile the number of nodes is $N$ and the number of edges is $M$. As the model mentioned above, the ASN is a type of undirected weighted network, so the weighted adjacency matrix can be used to express the ASN. The daily flights between sectors are considered as the weight of the edge and the ASN is expressed as

$$
B_{i j}= \begin{cases}f_{i j} & \text { if node } i \text { connects to node } j \text { with weight } f \\ 0 & \text { otherwise }\end{cases}
$$

where, in the ASN model, $B_{i j}=B_{j i}$ and $B_{i i}=0$ are satisfied.

The ASN model is a basis of complex network and it can be analyzed based on the general complex network theory. In the following section, some classical network methods are discussed.

\subsection{Degree distribution}

For a simple network with $N$ nodes, where $A_{i j}=A_{j i}=1$, the degree, denoted by $k_{i}$ is expressed as

$$
k_{i}=\sum_{j=1}^{N} A_{i j}
$$

Another method of expressing degree data is the cumulative distribution function

$$
P(k)=\sum_{k^{\prime}=k}^{\infty} P\left(k^{\prime}\right)
$$

where $P(k)$ is the cumulative degree distribution function and it indicates the probability distribution of nodes with degrees not less than $k$.

\subsection{Assortativity coefficient}

The assortativity coefficient of the network can be calculated to characterize the relationship between node degrees. The assortativity coefficient, denoted as $r$ is introduced by Pearson and it is defined as ${ }^{34}$

$$
r=\frac{M^{-1} \sum_{e_{i j} \in E} k_{i} k_{j}-\left[M^{-1} \sum_{e_{i j} \in E} \frac{1}{2}\left(k_{i}+k_{j}\right)\right]^{2}}{M^{-1} \sum_{e_{i j} \in E} \frac{1}{2}\left(k_{i}^{2}+k_{j}^{2}\right)-\left[M^{-1} \sum_{e_{i j} \in E} \frac{1}{2}\left(k_{i}+k_{j}\right)\right]^{2}},
$$

where $k_{i}$ and $k_{j}$ are the degrees of two nodes at the ends of an edge, $M$ is the total number of edges and $E$ is the set of edges. 
The range of the assortativity coefficient is $-1 \leq r \leq 1$. According to the assortativity coefficient, the relationship between node degrees is described as follows:

(i) In the case of $r<0$, the network shows disassortativity characteristics, namely large-degree nodes are more likely to link small-degree ones.

(ii) In the case of $r=0$, there is no correlation between node degrees. That is, nodes in the network are randomly connected and the expected neighbors of each node is constant.

(iii) In the case of $r>0$, the network shows assortativity characteristics, in which large-degree nodes are more likely to link large-degree ones.

\subsection{Degree correlation}

Degree correlation is an important statistical feature of the network, which describes the relationship between nodes with large degrees in the network and nodes with small degrees.

First of all, the average nearest-neighbor degree of node $v_{i}$ is defined as

$$
k_{n n, i}=\left[\sum_{j} a_{i j} k_{j}\right] / k_{i},
$$

where $k_{i}$ is the degree of node $v_{i}$ and $a_{i j}$ is the element of the adjacency matrix $A_{i j}$. Then, the nearest-neighbor average degree of a node with degree $k$ is expressed $\operatorname{as}^{35}$

$$
k_{n n}(k)=\sum_{k^{\prime}} k^{\prime} P\left(k^{\prime} / k\right)
$$

where $P\left(k^{\prime} / k\right)$ is the conditional probability that the edge belonging to a node with degree $k$ points to a node with degree $k^{\prime}$.

\subsection{Node strength correlation}

For a node $v_{i}$, the strength is defined as ${ }^{10}$

$$
s_{i}=\sum_{i=1}^{N} a_{i j} f_{i j},
$$

where $s_{i}$ is the strength of node $v_{i}$ and $f_{i j}$ is the weight of edge $e_{i j}$.

The average nearest-neighbor weight of node $v_{i}$ is defined as

$$
s_{n n, i}=\frac{\left(\sum_{j} f_{i j} s_{j}\right)}{s_{i}},
$$

where $s_{n n, i}$ is the average nearest-neighbor weight of node $v_{i}$. Then, the nearestneighbor average weight of a node with weight $s$, denoted by $s_{n n}(s)$ is

$$
s_{n n}(s)=\frac{\left(\sum_{i \mid s_{i}=s} s_{n n, i}\right)}{[N \cdot P(s)]},
$$

where $N$ is the number of nodes and $P(s)$ is the node strength distribution function. 


\subsection{Centrality measures}

The ASN is a complex network model, consequently, the centrality measures of each node can be analyzed by the complex network theory. According to the topological information, the centrality measures are as follows.

Degree centrality: The node degree is one of the basic statistical parameters of the complex network. The degree centrality, denoted by $C_{D}$ is a relatively simple centrality measure, which is defined as follows ${ }^{36}$ :

$$
C_{D}(i)=\frac{k_{i}}{N-1}
$$

where $k_{i}$ is the degree of node $v_{i}$ and $N$ is the number of nodes.

Betweenness centrality: The betweenness of node $v_{i}$ is defined as the fraction of shortest paths between node pairs that pass through the node of interest, which reflects the role and influence of the node in the entire network. The betweenness centrality is defined as $^{37}$

$$
C_{B}(i)=\sum_{s \neq t \neq i} \frac{\sigma_{s t}(i)}{\sigma_{s t}},
$$

where $\sigma_{s t}$ is the number of shortest paths between nodes $v_{s}$ and $v_{t}$, and $\sigma_{s t}(i)$ indicates the number of shortest paths between $v_{s}$ and $v_{t}$ which pass through node $v_{i}$.

Closeness centrality: The closeness centrality is based on the shortest path length between nodes. The centrality of such a node may be high, when the average path length between the node and others is relatively short. The closeness centrality of node $v_{i}$, denoted by $C_{C}(i)$ is expressed as ${ }^{38}$

$$
C_{C}(i)=\frac{N-1}{\sum_{j=1, j \neq i}^{N} d_{i j}},
$$

where $N$ is the number of nodes and $d_{i j}$ is the shortest path length between nodes $v_{i}$ and $v_{j}$.

Eigenvector centrality: The eigenvector centrality can consider the centralities of neighbors and is a relatively high version of degree centrality to a certain extent. Based on Refs. 39 and 40, the eigenvector centrality of node $v_{i}$, denoted by $C_{E}(i)$ is computed by

$$
C_{E}(i)=\lambda_{\max }^{-1} \sum_{j=1}^{N} a_{i j} \mu_{j},
$$

where $\lambda_{\max }$ is the maximum eigenvalue of adjacency matrix $A$ and $\mu=$ $\left[\mu_{1}, \ldots, \mu_{2}, \ldots, \mu_{N}\right]^{T}$ is the corresponding eigenvector.

\subsection{Node sorting algorithm}

In this section, a kind of node sorting algorithm is introduced based on the VCM. The VCM is a kind of objective weighting theory which uses the mathematical 
statistics method to analyze the processing of each physical structure centrality value (degree, betweenness, closeness and eigenvector centrality) to obtain the weight. In the evaluation index system, the index with larger difference in value is more difficult to achieve. Such indices that have a large impact on network physical characteristics will receive greater weight.

First, to determine the evaluation centrality measures, we can get the original matrix of $\left(r_{i j}\right)_{N \times H}$

$$
R=\left[\begin{array}{llll}
r_{11} & r_{12} & \cdots & r_{1 H} \\
r_{21} & r_{22} & \cdots & r_{2 H} \\
\vdots & \vdots & \vdots & \vdots \\
r_{N 1} & r_{N 2} & \cdots & r_{N H}
\end{array}\right]_{N \times H},
$$

where, $N$ is the number of items (i.e., nodes) to be evaluated and $H$ is the number of evaluation indices (i.e., centrality measures).

The original matrix $R$ is normalized, that is, the ratio of the column vector $r_{i j}$ in the matrix $R$ to the sum of all the elements in the matrix $R$ is taken as the normalization result. The formula is as follows:

$$
x_{i j}=\frac{r_{i j}}{\sum_{i=1}^{N} r_{i j}}, \quad j=1,2, \ldots, H,
$$

where, $x_{i j}$ is the element in the normalized matrix and the normalized matrix, denoted by $X$ is expressed as

$$
X=\left[\begin{array}{llll}
x_{11} & x_{12} & \cdots & x_{1 H} \\
x_{21} & x_{22} & \cdots & x_{2 H} \\
\vdots & \vdots & \vdots & \vdots \\
x_{N 1} & x_{N 2} & \cdots & x_{N H}
\end{array}\right]_{N \times H}
$$

Since the dimensions of the indices in the evaluation index system are different, it is not appropriate to directly compare the differences. In order to eliminate different effects of various dimensions, it is necessary to use the coefficient of variation of each node to measure the degree of difference in the centrality value of each node. The variation coefficient of each node is as follows:

$$
V_{j}=\frac{\sigma_{j}}{\bar{x}_{j}}, \quad j=1,2, \ldots, H,
$$

where $V_{j}$ is the variation coefficient of $j$ th centrality measure, also known as the standard deviation coefficient and $\sigma_{j}$ is the centrality measure standard deviation of node $v_{i}$. The weight of each centrality measure is

$$
W_{j}=\frac{V_{j}}{\sum_{j=1}^{H} V_{j}}
$$

where $H$ is the number of centrality measures. 
Finally, the comprehensive evaluation centrality value of each node is determined. The weights of the centrality measures are, respectively, multiplied by their corresponding measure values, and the evaluation model is

$$
C_{S}=\sum_{j=1}^{H} w_{j} x_{i j}, \quad i=1,2, \ldots, N,
$$

where $C_{s}$ represents the comprehensive evaluation function of each node.

Therefore, the final ranking values of nodes can be expressed as

$$
\left[\begin{array}{c}
C_{S}(1) \\
C_{S}(2) \\
\vdots \\
C_{S}(N)
\end{array}\right]=\left[\begin{array}{cccc}
x_{11} & x_{12} & \cdots & x_{1 M} \\
x_{21} & x_{22} & \cdots & x_{2 M} \\
\vdots & \vdots & \vdots & \vdots \\
x_{N 1} & x_{N 2} & \cdots & x_{N M}
\end{array}\right]_{N \times M} \cdot\left[\begin{array}{c}
w_{1} \\
w_{2} \\
\vdots \\
w_{H}
\end{array}\right],
$$

where $C_{S}(i)(i=1,2, \ldots, N)$ is the comprehensive sort value of node $v_{i}$.

\section{Topology Analysis of ASN}

The air sectors are the specific spaces in which aircraft operate, and their structural characteristics are critical to the safety and smoothness of air operations. Meanwhile, the analysis of sector topology characteristics is beneficial to the optimal configuration of airspace. According to the above analysis, the ASN is an undirected weighted model, so it can be analyzed using complex network theory and the results are as follows.

As shown in Fig. 3(a), the double-logarithmic relationship between cumulative node degree function $P(k)$ and degree $k$ is described. The relationship is divided into two phases. When the degree $k \leq 4$, the cumulative degree distribution obeys the power law distribution and the slope is -0.07 . When the degree $k>4$, the cumulative degree distribution also obeys the power-law distribution and the slope is -0.15 . As the node degree of the ASN is greater than the average degree, the number of nodes decreases rapidly, indicating that the network has a homogeneous structure feature to a certain extent. In general, the cumulative degree function of the ASN follows a two-regime power-law distribution.

It is shown in Fig. 3(b) that the cumulative node strength distribution $P(s)$ as a function of node strength $s . P(s)$ takes the logarithm, then the function has a linear relationship with $s$, indicating that the distribution follows the exponential distribution. Therefore, it can be seen that the flight flow between sectors is subject to a heterogeneous distribution.

From Fig. 3(c), we know the relationship between the nearest-neighbor average degree $k_{n n}(k)$ and degree $k$. In general they have a positive correlation and the slope is positive, that is, large-degree nodes are more likely to link large-degree ones. When $k>3$, the $k_{n n}(k)$ is close to a constant indicating that nodes with different degrees have a very similar neighborhood. In addition, the assortativity 


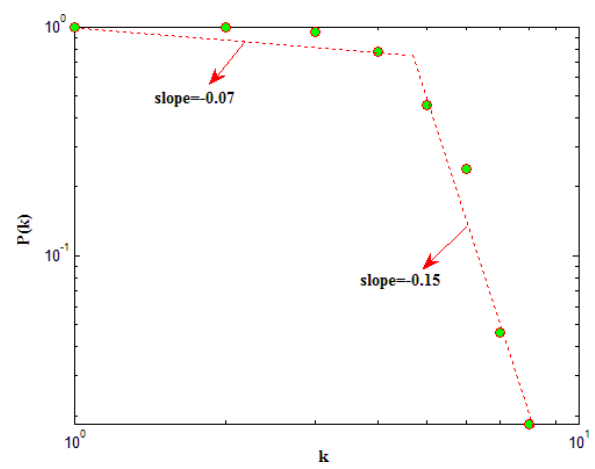

(a)

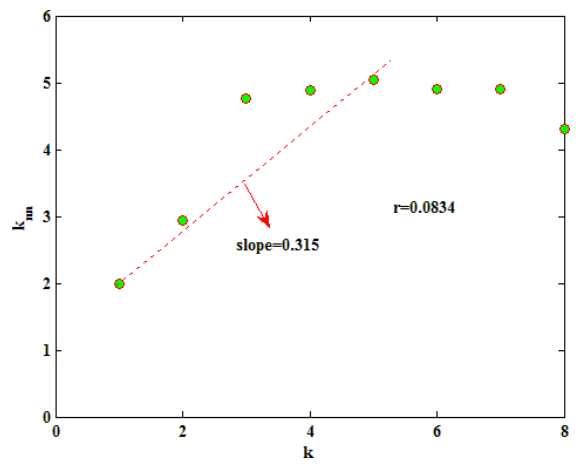

(c)

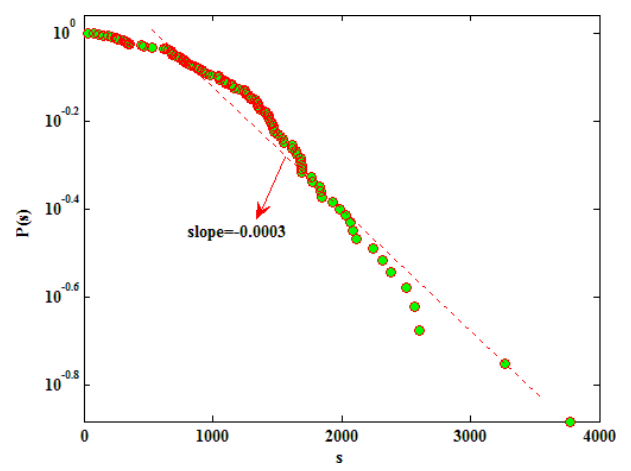

(b)

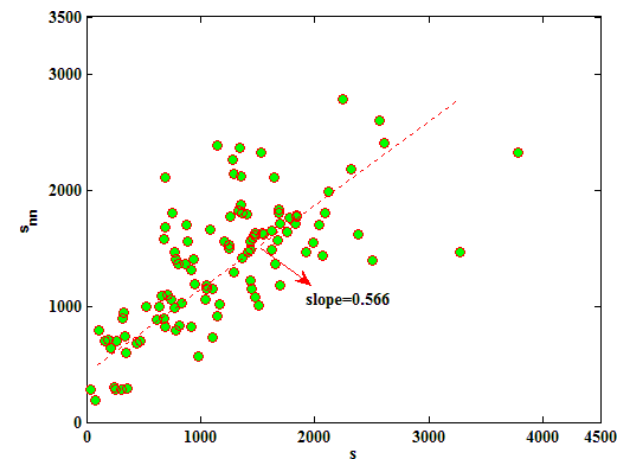

(d)

Fig. 3. (Color online) (a) The cumulative node degree distribution, (b) the cumulative distribution of node strength, (c) the node degree correlation and (d) the node strength correlation.

coefficient $(r=0.0834)$ of the ASN is positive. Therefore, to some extent, the ASN has assortative structure characteristics.

Figure 3(d) shows the relationship between nearest-neighbor average weight $s_{n n}$ and the node strength $s$. The slope is greater than zero, which means that they have a positive correlation. In a word, the results explain that the nodes with large strength tend to connect large-strength ones in the Chinese ASN.

The above content discusses the static structural characteristics of the ASN model, in the next, the relationship between the centrality of sectors and flights distribution is analyzed for the sake of reflecting the ASN characteristics well. The relationship between node strength and centrality values is described in Fig. 4.

Figure 4 depicts the relationship between node strength and different centrality values in the ASN model. In general, the node strength and different centrality values have positive correlation characteristics, that is, large-strength sectors are more likely to have large centrality values. Figure 4(a) shows that large-degree nodes tend to have large node strength, yet there also exists large-degree nodes with small strength. Simultaneously, Fig. 4(b) indicates the existence of nodes with high values of node strength with a low value of betweenness and most of the nodes 


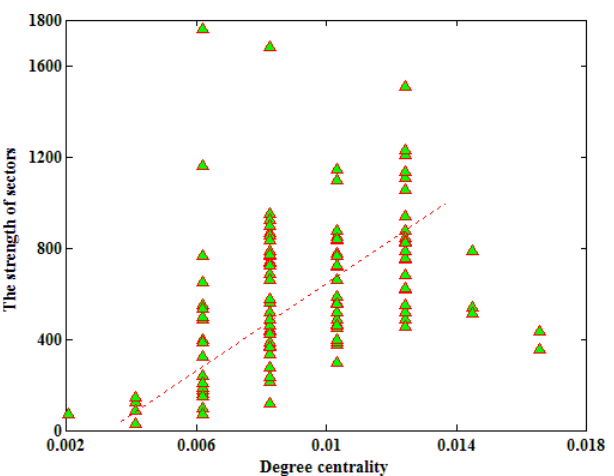

(a)

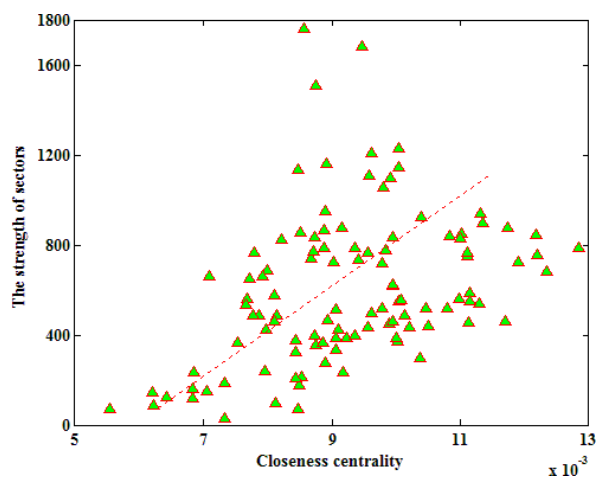

(c)

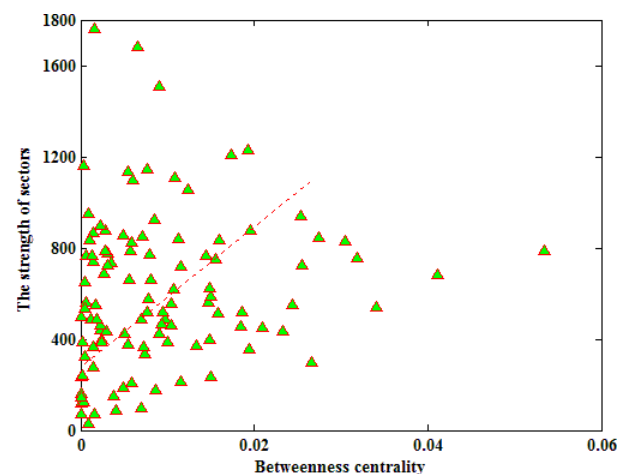

(b)

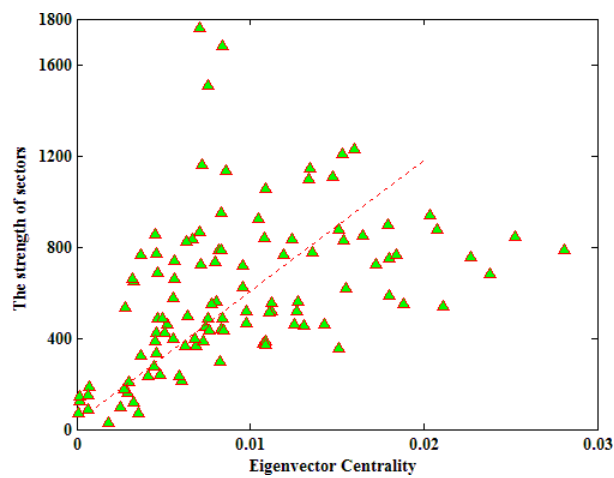

(d)

Fig. 4. (Color online) The relationship between node strength and different centrality values.

have a relatively small betweenness value. What is more, the positive correlations between node strength and closeness or eigenvector are more obvious [Figs. 4(c) and $4(\mathrm{~d})$ ]. Furthermore, most large-strength nodes have relatively large closeness or eigenvector values.

In summary, the topological properties of the weighted sector network are analyzed in this section by the complex network theory. This result helps us understand the physical structural characteristics of the airspace. Meanwhile, it also indicates that the traffic distribution in the sector has inhomogeneity and accumulation characteristics, and the sector with large centrality value tends to have a large traffic volume. To a certain extent, the above research provides a theoretical reference for further optimizing the airspace structure and identifying key sectors.

\section{Sector Ranking Analysis}

The key influences of the nodes in the ASN network are the degree, betweenness, closeness, eigenvector centralities, etc. Moreover, the influential nodes are 
also affected by the comprehensive effects of various attributes. According to the centrality measures in Sec. 3.5, the spatial distribution of the top-20 sectors in ASN is described in Fig. 5.

Figure 5(a) shows the top-20 nodes (green nodes) sorted through degree and the results are relatively scattered. Similarly, the distribution of the top-20 nodes based on the betweenness is also dispersed [Fig. 5(b)]. However, the results are relatively concentrated together, such as the top- 20 nodes based on the order of closeness and eigenvector centrality values [Figs. 5(c) and 5(d)], which show that the tightness of the sectors in central China is high.

Based on the above analysis, the VCM theory in Sec. 3.6 can be applied to obtain the weight of each centrality measure. Then, we can get the comprehensive ranking result of the sectors. The weight of each centrality measure is shown in Table 3 .

On the basis of Table 3, the weight values of the each centrality measures (degree, betweenness, closeness and eigenvector) are obtained. Then according to VCM, the comprehensive centrality value of the top-20 sectors is shown in Fig. 6 .

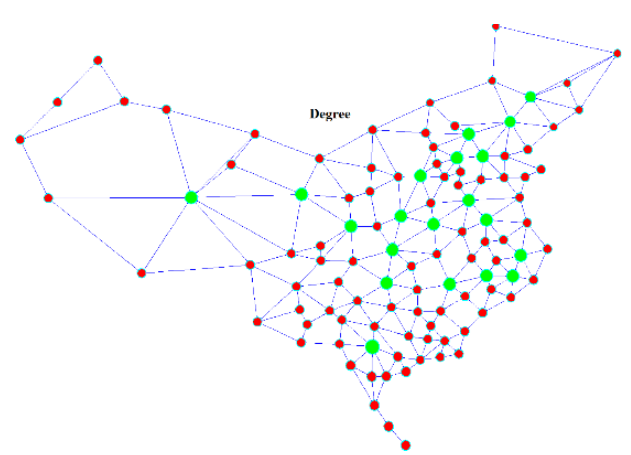

(a)

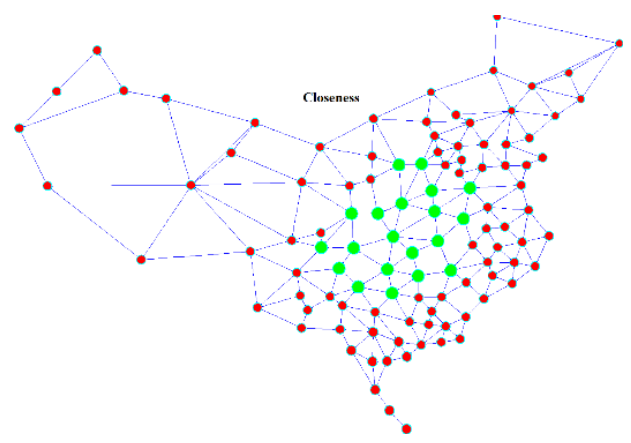

(c)

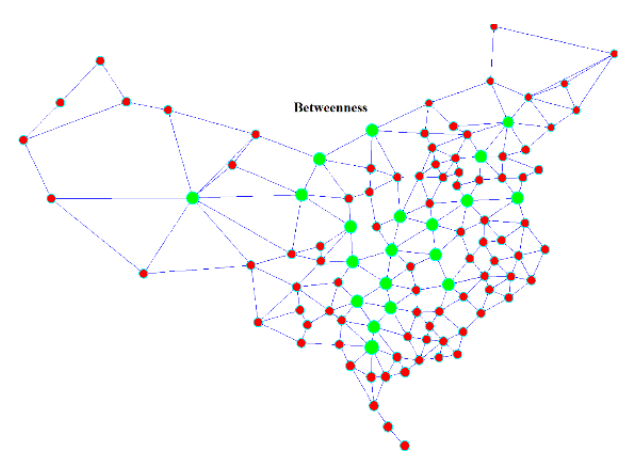

(b)

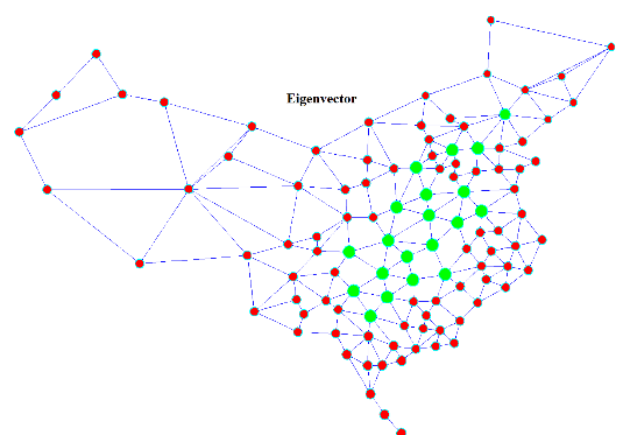

(d)

Fig. 5. (Color online) The spatial distribution of the top-20 sectors in ASN ranked by degree, betweenness, closeness and eigenvector centrality values. 
Table 3. The weight values of centrality measures.

\begin{tabular}{ccccc}
\hline Centralities & Degree & Betweenness & Closeness & Eigenvector \\
\hline Weight value & 0.138 & 0.488 & 0.075 & 0.299 \\
\hline
\end{tabular}

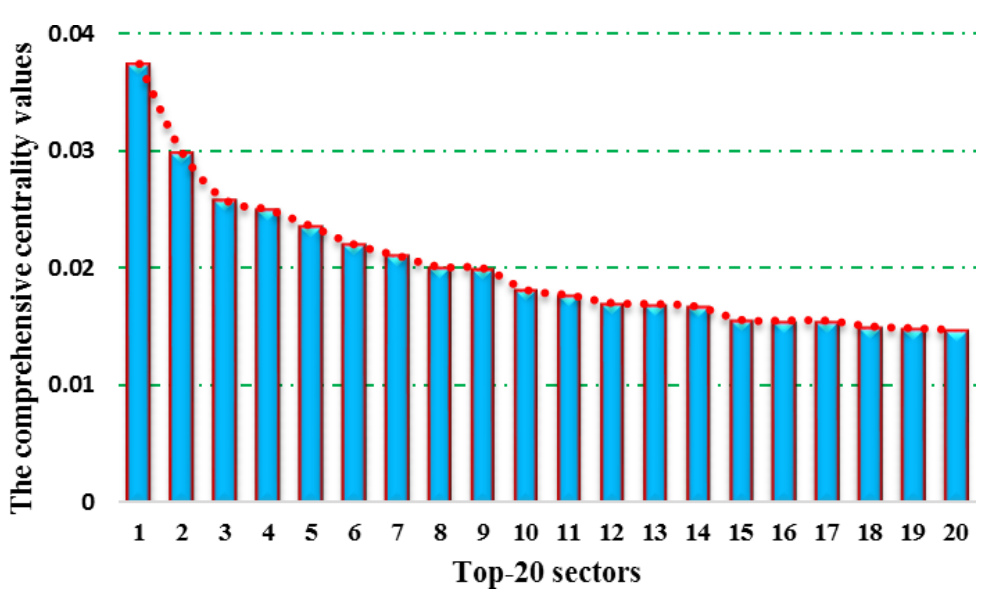

Fig. 6. (Color online) The comprehensive centrality value of the top-20 sectors by VCM (the geographical locations of the top-20 sectors are shown in Fig. 8).

For the results of Fig. 6 and other centrality ranking results, the next step is to verify the validity of each theory. As we know, the SIR model is a typical theory of infectious diseases and can be used to identify the influential nodes in complex networks. Then, the performance of different algorithms to identify influential nodes can be evaluated by the Kendall's tau coefficient. Therefore, in the following, we will introduce the SIR and Kendall's tau coefficient theories and make a comparative analysis on the effectiveness of VCM and other centrality measures.

SIR model. SIR can simulate the network propagation process and compare its results with the results of the proposed centrality measures. According to Ref. 41, there are three states in the SIR model, the susceptible $(S(t))$, the infected $I(t)$ and the removed $R(t)$. When the individual (node) is in an infected state, the neighbors who are susceptible to infection are infected with the probability of $\beta$, and the node of the infected state returns to the removed state with the probability of $\gamma$.

Kendall's tau coefficient: Suppose two random variables are $X$ and $Y$ (also can be seen as two sets), their number of elements is $N$. The Kendall's tau coefficient, denoted by $\tau$ is defined as follow ${ }^{42}$ :

$$
\tau=\frac{N_{c}-N_{d}}{0.5 N(N-1)}, \quad(-1 \leq \tau \leq 1),
$$

where $N_{c}$ and $N_{d}$ are the number of concordant and discordant pairs, respectively. If the consistency between the two rankings is perfect (i.e., the two rankings are the same), the closer the coefficient $(\tau)$ value is to 1 . 
In the process of simulation propagation, only one node in the ASN model is selected as the initial infected one in each step. Then, the susceptible neighbors will be randomly infected, and after a long period of time $(t)$, the infected nodes gradually decrease. The total number of infected nodes and removed nodes represented by time $t$, denoted by $F(t)$. The higher the value of $F(t)$, the more impact the node has. Furthermore, the spreading probability $\beta$ of SIR model is gradually increasing from 0.01 to 0.1 , and then the Kendall's tau coefficient $(\tau)$ is obtained by comparing the ranking list generated by different centrality measures and the ranking list generated by SIR model on the ASN. The results are shown in Fig. 7 .

Based on Fig. 7(a), in ASN, it is obvious that the proposed VCM method gives better performance than the other centrality measures. The performance of betweenness is second only to VCM for the line follows the VCM closely. Besides, the result for degree and the closeness centrality method is similar, because the line of degree is slightly higher than closeness. In addition, the eigenvector measure gives relatively poor performance. What is more, in Fig. 7(b), the higher the value of the Kendall's tau coefficient, the better the correlation between the method and the SIR model. The correlation line of VCM is the highest, therefore, it performs the best. Simultaneously, the correlation curves of degree and betweenness are slightly lower than the proposed VCM. Of course, the eigenvector follows closely closeness in the last place.

In a word, Fig. 7 shows that the proposed VCM method performs relatively better than others in identifying influential nodes. Furthermore, the simulation experiments indicate that the VCM centrality measure has validity and accuracy in ranking sectors to a certain extent.

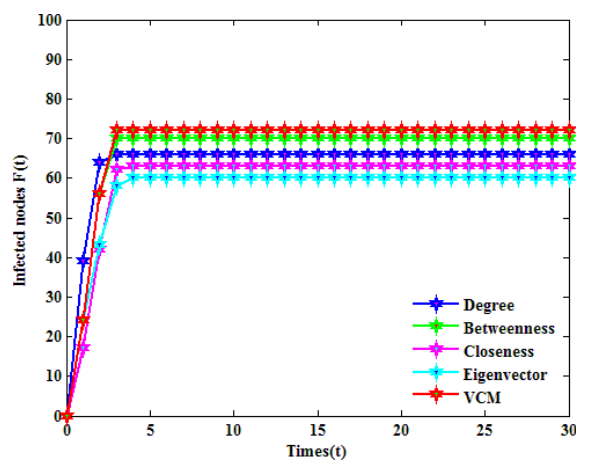

(a)

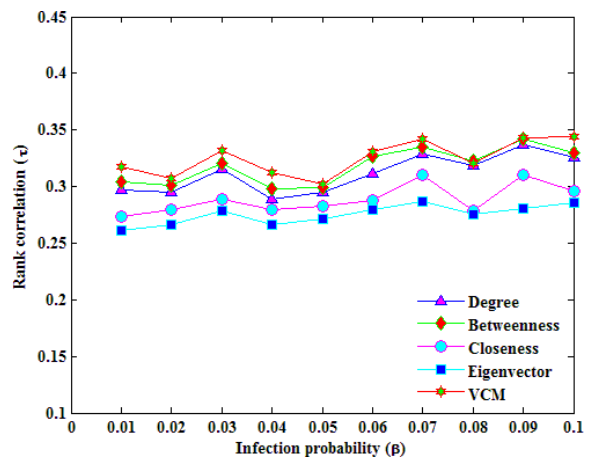

(b)

Fig. 7. (Color online) (a) The cumulative number of infected nodes $F(t)$ as a function of time ( $t=10$ and 100 implementations) in ASN with the initially infected nodes being those that either appear in the top-20 list by the proposed VCM, degree, betweenness, closeness or eigenvector centrality measures. (b) The Kendall's tau coefficient $\tau$ is obtained by the infection probability $\beta$ varying from 0.01 to 0.1 on the ASN and the results are obtained by over 100 independent runs (the results in Fig. 7 are the average value after about 100 simulations). 


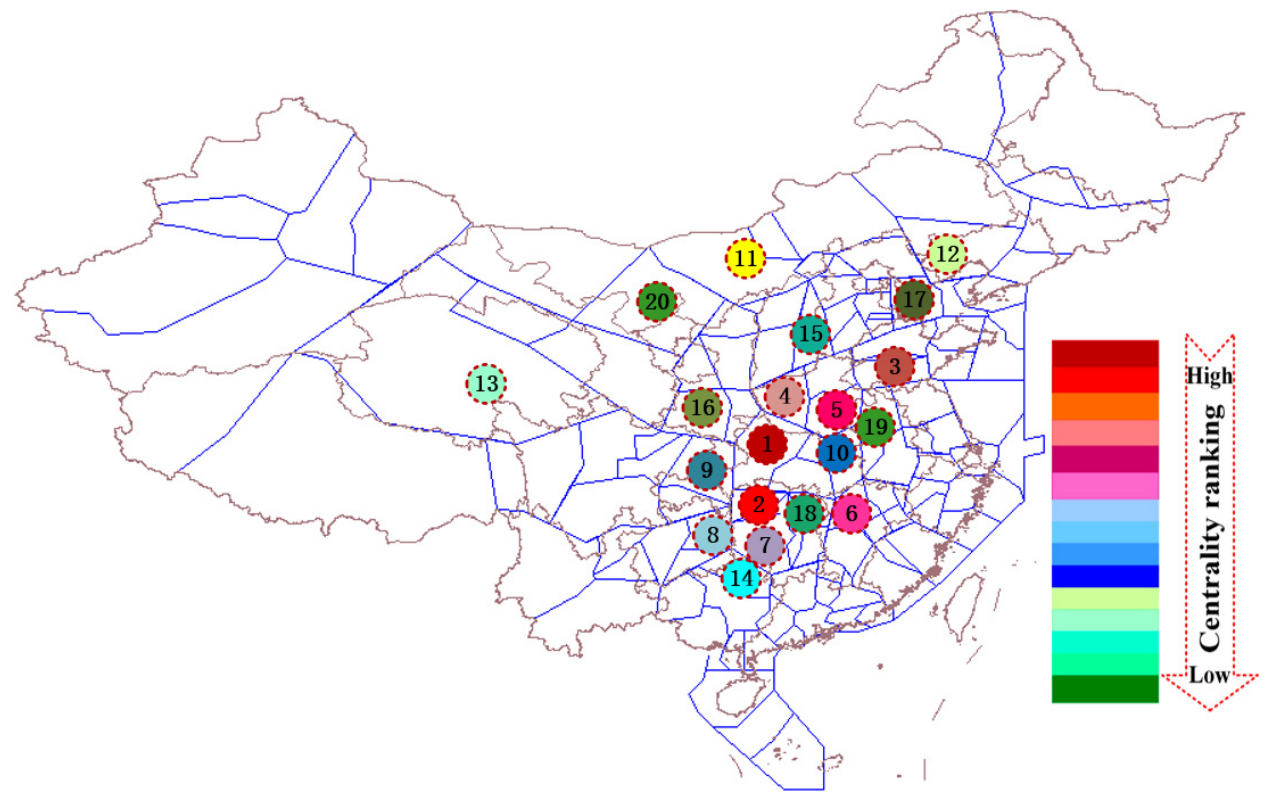

Fig. 8. (Color online) The spatial distribution of the top-20 sectors in ASN.

According to the above results, the spatial distribution of the top-20 sectors ranked by the VCM is analyzed, so we can understand the key areas of airspace operation. The distribution results are described in Fig. 8.

As depicted in Fig. 8, the top-20 sectors are expressed by the solid circles, where different colors represent different rankings. Besides, the ranking of top-20 sectors is also identified by numerical values. Geographically, the top-20 sectors are mainly distributed in the central and eastern regions of Chinese mainland. Of course, the distribution is consistent with the population density and the degree of aviation development. From the network structure, the distribution reflects the core area of the ASN, which has important guiding significance for the optimization and dynamic adjustment of the airspace.

In short, the distribution of the critical sectors reflects the bottleneck area of airspace operation to some extent. At the same time, it also defines the reference area for the treatment of air delays, which has certain reference significance for improving the efficiency of airspace operation.

\section{Conclusion}

In this paper, the air sectors of Chinese mainland is analyzed and then a weighted network model called ASN is built. The nodes of the ASN are the sectors and air routes between sectors are the edges, meanwhile, the flights between nodes are the weight of the edges. Then, a node sorting theory is proposed based on the VCM. Next, the topological features of the ASN are discussed by complex network theory 
and the key sectors are ranked by the proposed theory. The conclusions are as follows:

(i) The topological properties of the weighted ASN is analyzed, whose result helps us to understand the structure characteristics of airspace. Meanwhile, it also indicates that the traffic distribution in the sector has inhomogeneity and accumulation features.

(ii) The VCM is not limited to only one centrality measure, but considers different ones. In other words, different centrality measures (degree, betweenness, closeness and eigenvector) as the multi-attribute of complex network in VCM application. Consequently, the coefficient of variation of each centrality index can effectively measure the difference in the value of each centrality measure.

(iii) The key air sectors of ASN are ranked by the proposed VCM theory and the results are verified through the SIR model and Kendall's tau coefficient, indicating that the VCM centrality measure has validity and accuracy in ranking sectors to a certain extent. Simultaneously, the result is useful for finding sectors that are prone to congestion and providing some theoretical references for optimizing the structure of sectors and improving the efficiency of airspace.

In the future, we will further study the traffic congestion mechanisms of the air sectors, as well as the influence relationship between the air route network and the ASN, and further exploratory approach to reduce air delay.

\section{Acknowledgment}

This work is supported by the National Natural Science Foundation of China (CN) (Grant Nos. 71731001 and 61501225).

\section{References}

1. O. Lordan et al., J. Transp. Geogr. 37, 112 (2014).

2. M. Zhang et al., Chaos Solitons Fractals 112, 97 (2018).

3. D. S. Dunn and D. S. Wilkinson, Transp. Res. E 90, 39 (2016).

4. T. Verma et al., Int. J. Mod. Phys. C 27, 1650141 (2016).

5. T. Verma, N. A. M. Araújo and H. J. Herrmann, Sci. Rep. 4, 5638 (2014).

6. J. Lin and Y. Ban, Physica A Stat. Mecha. Appl. 410, 302 (2014).

7. Reynolds-Feighan, J. Air Transp. Manage. 16, 109 (2010).

8. O. Lordan and J. M. Sallan, Chin. J. Aeronaut. 30, 554 (2017).

9. M. Hossain et al., Australian airport network robustness analysis: A complex network approach, in Proc. Australasian Transport Research Forum, 2-4 October (Brisbome, Australia, 2013), pp. 1-21.

10. W. B. Du et al., Physica A Stat. Mech. Appl. 465, 579 (2017).

11. W. B. Du et al., Transp. Res. E 89, 108 (2016).

12. L. Dai et al., J. Transp. Geogr. 69, 271 (2018).

13. W. Du et al., Chin. J. Aeronaut. 30, 330 (2017).

14. X. Sun et al., J. Transp. 170, 46 (2017).

15. G. Gurtner et al., PLoS One 9, e94414 (2014). 
16. S. Roy, M. Xue and B. Sridhar, Vulnerability metrics for the airspace system, in 12th USA/Europe Air Traffic Management $R$ and D Seminar (2017).

17. P. Wei, G. Spiers and D. Sun, Transp. Res. E 61, 13 (2014).

18. O. Lordan et al., Transp. Res. E 68, 155 (2014).

19. X. Sun, S. Wandelt and F. Linke, Transp. B, Transp. Dyn. 3, 153 (2015).

20. M. G. Song and G. T. Yeo, Asian J. Shipp. Log. 33, 117 (2017).

21. J. Jiang et al., Int. J. Mod. Phys. B 31, 1750186 (2017).

22. X. Sun, S. Wandelt and X. Cao, Netw. Spat. Econ. 17, 1 (2017).

23. X. Zhang et al., Knowl. Based Syst. 42, 74 (2013).

24. M. M. Tulu, R. Hou and T. Younas, IEEE Access 6, 7390 (2018).

25. D. B. Chen et al., PLoS One 8, e77455 (2013).

26. Q. Ma and J. Ma, Physica A, Stat. Mech. Appl. 465, 312 (2017).

27. Y. Du et al., Physica A, Stat. Mech. Appl. 399, 57 (2014).

28. T. Bian and Y. Deng, Chaos 28, 043109 (2018).

29. A. Zareie, A. Sheikhahmadi and A. Fatemi, Chaos, Solitons Fractals 104, 485 (2017).

30. S. Wang, Y. Du and Y. Deng, Commun. Nonlinear Sci. Numer. Simul. 47, 151 (2017).

31. T. Bian, J. Hu and Y. Deng, Physica A, Stat. Mech. Appl. 391, 1777 (2017).

32. J. Tang, L. Khoja and H. R. Heinimann, Modeling stock survivability resilience in signed temporal networks: A study from London stock exchange, in Int. Workshop Complex Networks 8 Their Applications 689(2), 1041 (Springer, Cham, 2018).

33. K. C. Pien et al., Transp. A, Transp. Sci. 11, 772 (2015).

34. M. E. Newman, Phys. Rev. Lett. 89, 208701 (2002).

35. R. Pastorsatorras, A. Vázquez and A. Vespignani, Phys. Rev. Lett. 87, 258701 (2001).

36. G. J. Ren et al., Chin. J. Phys. 57, 382 (2019).

37. L. C. Freeman, Sociometry 40, 35 (1977).

38. G. Sabidussi, Psychometrika 31, 581 (1966).

39. P. Bonacich and P. Lloyd, Social Netw. 23, 191 (2001).

40. S. P. Borgatti, Social Netw. 27, 55 (2005).

41. R. M. Anderson and R. M. May, Infectious Diseases of Humans, Vol. 1 (Oxford University Press, Oxford, 1991).

42. M. G. Kendall, Biometrika 33, 239 (1945). 